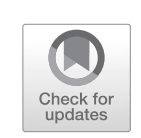

\title{
The Global Trajectories of Compulsory Education: Clustering Sequences of Policy Development
}

\section{Fabian Besche-Truthe}

\section{Introduction ${ }^{1}$}

Compulsory education became an imperative trait of sound state education systems at the very latest with the Universal Declaration on Human Rights (UN General Assembly 1948, Article 26, 1). Ever since the Education for All Dakar Framework for Action, in which the members of the United Nations Educational, Scientific and Cultural Organization (UNESCO) committed to ensuring access to "free and compulsory primary education" $(2000,8)$, a nation-state without compulsory education has hardly been imaginable. In fact, only a handful of United Nations

\footnotetext{
${ }^{1}$ This chapter is a product of the research conducted in the Collaborative Research Center "Global Dynamics of Social Policy" at the University of Bremen. The center is funded by the Deutsche Forschungsgemeinschaft (DFG, German Research Foundation)—project number 374666841SFB 1342.
}

F. Besche-Truthe $(\bowtie)$

Collaborative Research Centre 1342 "Global Dynamics of Social Policy", University of Bremen, Bremen, Germany

e-mail: fbesche@uni-bremen.de 
(UN) members do not have a compulsory education law in place; with Solomon Islands, Oman, and Bhutan being counted among them. Seeing as the world is pledged to Education for All, universal primary education (mentioned in the Millennium Development Goals (MDGs)), and universal secondary education (mentioned in the Sustainable Development Goals (SDGs)), one can assume that making education mandatory would constitute a viable first step toward achieving these goals. In accordance with the SDGs, extending the duration of compulsory education would be the second step. The extension can be targeted by either raising the school-leaving age or lowering the school-entry age, thus making preprimary education compulsory.

While the social sciences agree on the value of a sound education system, the focus of international comparative research has been on the determinants of the spread or retention of specific educational structures across the globe (Busemeyer and Trampusch 2011 provide a good overview). Causes relating to education system transformation have also been extensively analyzed. In this chapter, I take a step back and look at the larger picture, the constitutive factor of a state education system: compulsory education laws. In contrast to past studies, I aim to explore the development of compulsory education and its duration over time. How did policies concerning compulsory education develop globally? Are any trends discernible? Are there clusters of countries that develop similarly regarding compulsory education legislation?

Starting with the premise that there are different ideas about the necessary duration of education, compulsory education policy constitutes an observable manifestation of the importance of state-led education. Moreover, recognizing that these views are changing with time, ideas manifest themselves within policy changes. Therefore, research should spotlight the very changes over time. The policy developments grant insight into how compulsory education evolved in past decades. Finding common developments in nation-states might support further research on other policy developments and highlight commonalities previously unseen. As described below, past and current scholarship, regardless of theoretical and methodological background, focus on either the origins or outcomes of policies. Using snapshots or predefined outcome 
variables, these studies disregard developments that unfold over long stretches of history.

Drawing on recent developments in Sequence Analysis (SQA), this study seeks to depict and analyze the trajectories of compulsory education policies in 167 countries from 1970 to 2020. SQA lends itself as a method of inquiry because it enables the researcher to regard the whole trajectory of policy development as a single unit of analysis. Thus, it allows us to draw upon and explore the temporal context of policy development. Here, context becomes a point of entry for thinking about how processes of change relate to one another in an environment that unfurls over extended periods of time (Pierson 2004, 172). This opens avenues previously untrodden and allows one to ask new questions, that is, how did policy develop, and how is that different from the same policy in a different location at distinct times? How can we study dynamics macroquantitatively without losing the focus on the temporal and spatial context? How can this be accomplished with as little predetermination as possible? I utilize recent methodological developments for an inquiry that is, both, broad across time and space as well as narrow in analyzing the development paths. My chapter yields the first exploration into different trajectories of the duration of compulsory education and how these trajectories can be subsumed and clustered into specific development paths.

The chapter continues with a short overview of the current state of compulsory education legislation and summarizes potential explanatory theories that are widely used in education policy research. After presenting recent empirical evidence on the global expansion of compulsory education, I make the case for a descriptive rediscovery of changing compulsory education policies. A brief introduction into the method of SQA is followed by a detailed description of different trajectories of compulsory education policies. Furthermore, a clustering of similar trajectories based solely on the data and calculated with an Optimal Matching algorithm is presented. In the end, I provide an initial explanation regarding these different trajectories and conclude with an outlook regarding the focus of future research. 


\section{The State of Compulsory Education Policy}

Even before the two World Wars, education was seen in the Western world as a salvation for a myriad of social, political, and economic problems. In Prussia, compulsory education was introduced because of the need for a polis that fit with the hierarchical structure of society as well as a military that was able to sufficiently follow orders. In line with the Pietist puritan tradition, the young United States of America introduced compulsory schooling state by state, starting in New England (Rickenbacker 1999). In the Ottoman Empire, compulsory education was introduced in 1869 and was intended to build a coherent Ottoman culture and populace (Cicek 2012).

On the other hand, former colonies quickly introduced compulsory schooling after the surge of independence-some even before formal independence. In Ghana, for example, the legislative Assembly of 1951 "declared basic education to be free and compulsory for school-aged children" (Marlow-Ferguson 2002, 506), even though Ghana only became officially independent in 1957. In this 'new era', education was seen as embodying the ideals of a caring and prosperous state. Education became a human right and compulsory education an obligation for the nationstate. This is evident in the Bolivian entry into compulsory education; for example, the Movimiento Nacionalista Revolucionario introduced compulsory education as an attempt to reach new generations in their endeavor to build a truly democratic republic (Marlow-Ferguson 2002, 72).

Compulsory education is a very divisive political issue. Changes are difficult to adopt on the policy level and even harder to implement on the ground. Political struggles revolve around ethical questions (Hodgson 2016; Tan 2010) as well as budgetary issues. Extending mandatory schooling drains governments' monetary resources. That is why, in India, an extension of the right to free and compulsory education from the ages of six to fourteen to the ages of three to eighteen has been dropped from a recent education policy change, specifically because of the "financial burden" the state would have to bear (Dutta 2019). A state that has weak institutional capacities might also have a hard time monitoring whether 
the duration of compulsory schooling is being fulfilled, especially in rural areas.

\section{Explanatory Attempts for Compulsory Education Policymaking}

Having just explored historic and current developments of compulsory education policies, it is now time to focus on theories that try to explain the convergence of education policies. In line with this edited volume, the theories presented here build the canon of explanations on the development of 'modern' education systems. They either emphasize ideational influences, as evident in neo-institutionalist accounts (e.g. Ramirez 2013) and global economic influences, as shown in the World System approach (e.g. Griffiths and Imre 2013), or a mixture of both while highlighting specific national factors, as carried out in Cultural Political Economy (e.g. Dale 2000).

Proponents of the isomorphism thesis (Chap. 1) can be subsumed under the general headline of neo-institutionalists. When considering the global convergence of state structures, they highlight the normative influence of a World Culture pressuring policymakers to implement seemingly rational institutions. Neo-institutionalist theory argues that education policies spread around the globe through globally theorized models of 'what is a problem that needs to be solved' and by defining the worth of the 'good' salvation for that problem (Strang and Meyer 1993). The spread of idealistic norms has culminated in an impressive isomorphism of state structures and policies not predicted by other theories. Yet, "world cultural models are highly idealized and internally inconsistent, making them in principle impossible to actualize" (Meyer et al. 1997, 154). Thus, the theory acknowledges the phenomenon of decoupling, leaving some room for diverging policy developments.

However, neo-institutionalist explanations lack an engagement that goes beyond the sole valuing of some abstract model of education. In a volume edited by Anderson-Levitt (2003b) many anthropological case studies find a common model of schooling, for example, the so-called 
egg-carton school (Anderson-Levitt 2003a, 6) but they also show that these models are actually lived, experienced, and built in very different ways according to the (sub-)national contexts. Although World Culture theory claims to describe diffusion processes of abstract outcome variables quite well, it ignores probable conflating mechanisms and time inconsistencies in policy adoption (Dale 2000). Time, as a variable, gets degraded to specific structural frameworks, whereas norm development becomes universally influential, impacting policies with increasing rapidity. Variation in national institutional evolution, including the speed, order, and sequencing of it, is seen to starkly diminish since World War II (Boli et al. 1985).

Another approach analyzing the convergence of state structures can be subsumed under the headline of realist approaches, which culminated in the World System approach. It assumes political units are tied together in a world system grounded in capitalism (Wallerstein 2005, 24). This system is divided into the core, semi-periphery, and periphery. Membership of countries in one of these spheres dictates the possibilities of policymaking because of the divergence of valuable production processes along with the capital capabilities emerging from that. From this view, economic inequalities determine specific modalities of " $[s]$ chooling, rather than serving the interests of the majority in the periphery, abets the process of capital accumulation by hegemonic actors" (Arnove 2009, 105). Global isomorphism might be inhibited through the different positions in this system. However, widening the agenda to also consider dependencies and hegemonic coercion, the World System approach leaves little room for intragroup differences on paths toward similar outcomes. Moreover, the theory is depicting a widespread institutional stasis in which most countries are locked in their position. This translates to a static policy arena in which, if ever, changes are rare and should overlap depending on the distance to the core.

Contrary to the purely constructivist and Marxist-oriented approaches, Cultural Political Economy (CPE) attributes changes in policy to a multitude of factors, including changing political and economic parameters (Dale 2000). Using a CPE framework, Toni Verger et al. (2016) focus specifically on developmental paths. The authors find that although global discourse on education privatization is surging and has to some 
extent, elevated into a global norm, processes constituting the privatization and policy outcomes "can be extremely diverse in nature" (Verger et al. 2016, 148). The authors detect six different paths toward privatization. Thus, on the surface, seemingly congruent policy developments are comprised of polylithic trajectories that are influenced by different agents as well as different ideational, economic, and political rationales. The goal of the book by Verger et al. is an in-depth analysis of the different clusters of trajectories that led to education privatization. I build on that framework to quantitatively explore the routes compulsory education policy took in different locations around the world over 50 years.

All the approaches mentioned explain some similarities and dissimilarities of state education systems. Nonetheless, research has been mainly limited to a focus on either the origins, the output, or the outcome of education policy. Attempts at answering why nation-states initially required children to receive an education largely ignored differences in timing, sequencing, and the determining factors inherent to this contextual view. Research focuses on 'why' policies were implemented in the first place (e.g. Meyer et al. 1997; Wallerstein 2005). In this regard, event history approaches, although taking duration until an event seriously, have the caveat of a teleological focus, that is, the introduction of one (abstract) policy. While Seitzer, Besche-Truthe, and Windzio (Chap. 2) investigate the influence of cultural similarity regarding the introduction of compulsory education, the analysis stops at one point in time. Those attempts focus on a decisive answer on 'when' policy adoptions happen. Still, the method, by design, leaves blind spots, especially the possible steps taken until a predefined end-state is reached and focuses on one transition. Hence, it cannot answer the question of what happens after an initial adoption, and, since the method is focused on rather abstract policy determinants, it cannot answer exactly 'what' was adopted.

In outcome-centered research, duration of compulsory education is used as an independent variable in assessing the influence of longer schooling on enrollment and attendance (e.g. Landes and Solmon 1972), dropout rates (Diaz-Serrano 2020), poverty reduction (Zhang and Minxia 2006), fertility (Wilson 2017), health (Courtin et al. 2019), and so on (see also Stephens and Yang 2014 for a myriad of effects). 
Precise investigations of the institutional change of compulsory education are a rare endeavor. When conducted, qualitative case studies account for the majority. These illustrate the highly conflictual nature of compulsory education laws. For instance, in the year 2000, after almost thirty years of denying the necessity of any law, the Singaporean government quite suddenly argued for ten years of compulsory education (Tan 2010). However, due to pressure from home-schooling parents and the Malay/Muslim community, attending mostly madrasah schools, compulsory education was finally introduced in 2000 with a duration of six years. Accordingly, Hodgson (2016) illustrates the remarkably ethical discourse regarding a proposed increase in duration within Western Australia. The policy discourse on extending the school-leaving age was highly idealistic and hinged on neoliberalist themes (Hodgson 2016, 502-503). Similar discourses, highlighting the need for longer education, especially for young people at risk, can be observed in a number of Western countries starting from the 2000s (Hodgson 2016, 495ff).

Contrary to the case studies, Murtin and Viarengo (2011) analyze factors influencing the expansion of compulsory education from 1950 to 2000 in fifteen European states. They detect strong evidence for betaconvergence: Countries with lower initial values extended the duration more than those with higher initial values. The most statistically compelling reason for the general trend of extending compulsory education is trade openness (Murtin and Viarengo 2011, 505). This does hold true for the investigated Western European cases, but leaves open whether the same mechanism can be detected in post- or nonindustrialized countries.

While Murtin and Viarengo (2011) focus on the role of economic influence when it comes to the expansion of compulsory education in postwar Europe, Chapter 2 introduces the connection of event history analysis with a relational approach. Nonetheless, empirical attempts like the ones just mentioned use models that compare country-years and not country-trajectories, leaving the specific developments per country unattended. They might give answers to 'what' policies were adopted as well as 'why' and 'when' those were adopted, but they are unable to discern 'in which order' changes happened. Only a focus on the developmental paths enables one to combine the juxtaposition of the origin, output, outcome, and the very steps that lead or decisively did not lead to the 
state currently observed. This helps to recognize that any process is environed by its temporal location, its place within a sequence of occurrences (Pierson 2004, 172).

With this chapter, I aim to analyze compulsory education policy outlined in a way that takes sequences of change serious and, thus, investigates choices of policymakers around the globe structured through the progress of time. In this approach, policy outlines are not seen deterministically but due to structural factors like timing, order, and sequencing, display much more contingency than assumed by classic social science (Howlett and Goetz 2014, 480). In the words of Pierson (2004, 172), I want to know not just 'what' the duration of compulsory education was and 'when' change occurred but 'in which order' changes were made. As an additional feat, I am able to see 'for how long' the state of one policy was static and not changing at all.

\section{Methodological Remarks}

"For many years, our usual approach in sociology has been to think about cases independent of one another and, often, of the past" (Abbott 1995, 94). According to Abbott, empirical research erases the stories behind social reality by focusing on causality based on some variables' manifestations. Contingent narratives become impossible (Abbott 1992, 429). However, this does not apply to all research. There have been remarkable attempts at describing the emergence of compulsory education laws and the expansion of education opportunities, which take the past and specifically social action into account. This action is either intentionalmotivated first and foremost by economic situations and interdependencies as in a capitalist World System — or unintentional — motivated by institutionalized ideals in a World Culture. However, these theories have been starkly criticized by empirically oriented researchers for lacking causal variable-oriented applications. These critiques are correct in their assessment but wrong in their solutions. What is missing in current research, especially in political science, is a thorough description of policies, their histories, and evolutions. Although not a new methodology, it is worth mentioning the unique techniques of Japanese comparative education. In 
their view, the primary focus should be on the description of unique features within a given area of study "rather than the discovery of 'universal' laws and theories" (Takayama 2015, 39).

By placing compulsory education policy as the focal point, I aim to analyze the policy's trajectories globally. In SQA, the unit of analysis is the sequence itself. This goes further than a time-series, cross-section model because I do not rip apart yearly observations of compulsory education durations nor try to correlate yearly measured variables on these dependent values. Event History Analysis takes time seriously, especially process time until an event. Unfortunately, it is focused only on a transition from one category to another. It thereby leaves more complex steps unattended. Instead, I take the whole trajectory of compulsory education duration as one case. The aim is to first separately describe the trajectories of the duration of compulsory education for a large number of countries and then to search for patterns of similar trajectories. Through the recontextualization, that is, regarding institutional change not as single incidents but the focus on the very development of one policy, I am able to answer questions, previous research was unable to ask: In which order did the extension of compulsory education occur? When were critical junctures that changed the outlook of compulsory education? How is a policy developing not in one country but in relation to other countries as well as to time? Hence, I argue that it is important 'when' institutionalization of a specific duration of compulsory education occurs. It is furthermore important to investigate how these embedded aspects interact with the broader social context of other nation-states' policy developments (see Pierson 2004, 77f). Analyzing sequences allows me to identify linkages between processes in distinct spaces and at distinct points in time.

My dataset contains the number of years of compulsory education as defined in the legal framework. In building the dataset, I started with data from the UNESCO Institute for Statistics (UIS), where more historic data have been added from a variety of sources. ${ }^{2}$ In total, the dataset covers 167 countries over a time span of fifty years from 1970 until 2020. In-depth analyses of certain cases illustrate difficulties regarding sources

\footnotetext{
${ }^{2}$ I am especially grateful to my research assistant Philip Roth for helping to compile this vast and encompassing dataset of which I only use a small amount.
} 
and the correct definition of compulsory education. For instance, while the UIS website describes the compulsory education in Guatemala as encompassing all children aged $0-15$, which would result in sixteen years of compulsory education, the International Bureau of Education (2011) states that compulsory education consists of 3 years preprimary, 6 years primary, and 3 years secondary education; culminating in twelve years. The latter value has finally been coded. In case of uncertainties for countries with state responsibility over education, for example, Canada, the coding has been oriented on the law established for the most populated state or the capital. In any case, it tries to mirror what UNESCO reports on the federal nation-state. The data are available in the Welfare State Information System (https://wesis.org).

As previously noted, I am not interested in the specific time point of the policy's first introduction, but rather in the trajectory. Thus, in the following analysis, I start my inquiry after a surge of former colonies gained independence for more consistency. The data were recoded into sequences using the $R$ package TraMineR (Gabadinho et al. 2011): "[S] equences are made of three basic dimensions: the nature of the successive states, chosen among the alphabet; the order in which they occur; [and] their duration, that is, the duration of constant subsequences" (Blanchard 2011, 4 emphasis in original). In my case, the nature of the state is the number of years of compulsory education. The sequences are ordered according to successive years in calendar time. Changes in the duration of compulsory education have been coded according to the de jure implementation of the policy.

\section{Sequences of the Duration of Compulsory Education}

Figure 3.1 shows all sequences ordered by their starting value in the year 1970. The horizontal lines, each represents one nation-state and the colors show the valid duration of compulsory education in that specific year. One can see that a few sequences show no change in their duration. These countries have a stagnant trajectory. On the other hand, some seem very 


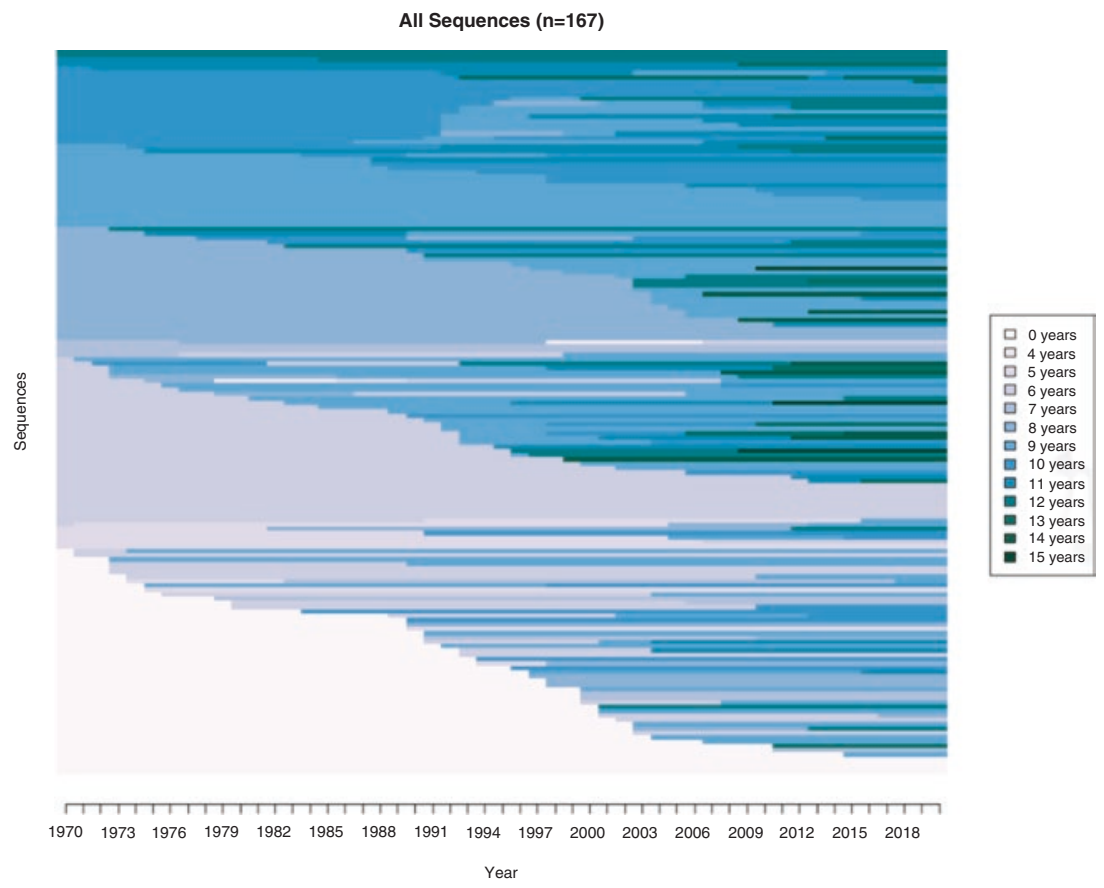

Fig. 3.1 All sequences of the duration of compulsory education policy

active in policy changes. One can discern a general trend toward more years of compulsory education. However, timing and actual extension, that is, by how many years, is difficult to systematize with this first largescale observation.

To provide a better overview and a first glance at different trajectories, Figs. 3.2 and 3.3 show the sequences grouped by income group and cultural spheres, which have been used in Chap. 2. Following economic arguments, I would suspect stark differences between income groups and coherent trajectories within a group. If a World Culture is influential, I would suspect no discernible differences in the economic as well as in the cultural spheres.

Through an economic grouping of the trajectories, one can see that although some countries never changed the duration of their compulsory schooling, a dynamic is detectable. General differences become a bit 

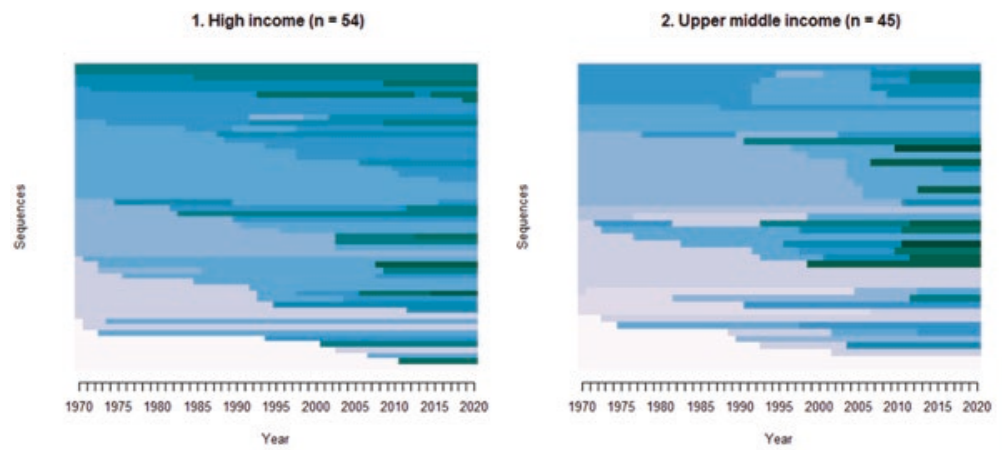

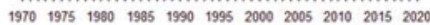

Year
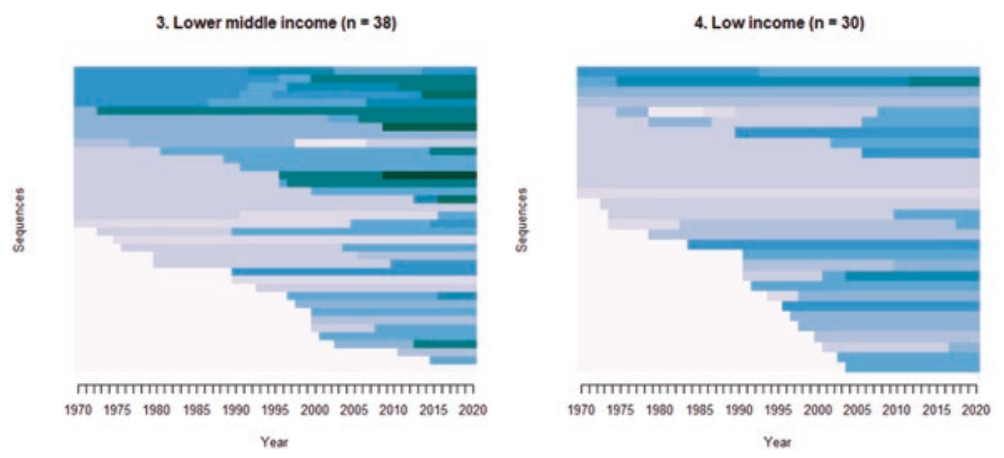

Fig. 3.2 Sequences grouped by income classification according to the WDI by the World Bank

more discernible when looking at different income groups. However, there is remarkable variance in the four different groups as defined in the World Development Indicators (WDI). The durations range from five to fifteen years in lower middle-income countries and from six to fourteen years in high-income countries. One can detect on average, highincome countries have longer durations of compulsory schooling with a mean of around 11 years. In contrast, low-income countries have around eight years of compulsory education on average. The largest differences are detectable in the middle-income groups, in which we see a similar pattern of average durations in upper middle-income countries, which have higher values than lower middle-income groups. Interestingly, there are countries surpassing high-income countries in duration with fourteen to fifteen years of compulsory education. These high values are possible 


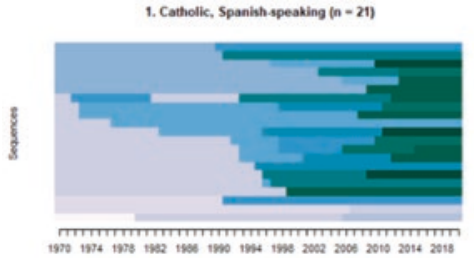

rear

3. Easter Europe (* VNM, UGA) (n - 19)

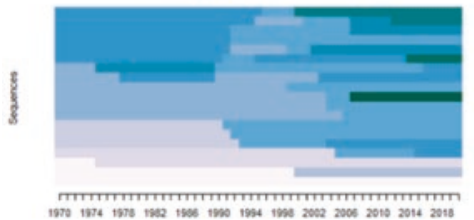

Year

5. dominantly Muslim ( $n-42)$

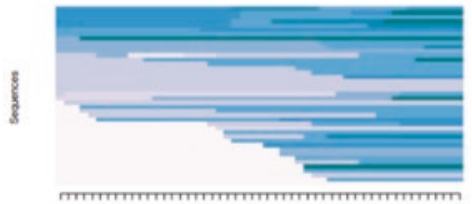

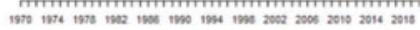

rear

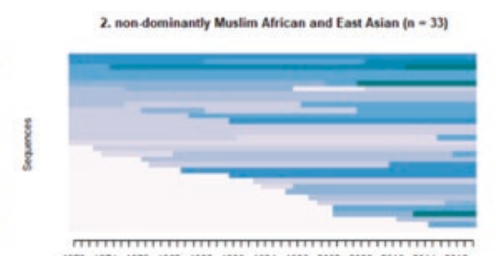

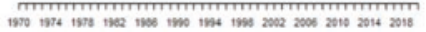

4. WEIRD $(n-4)$

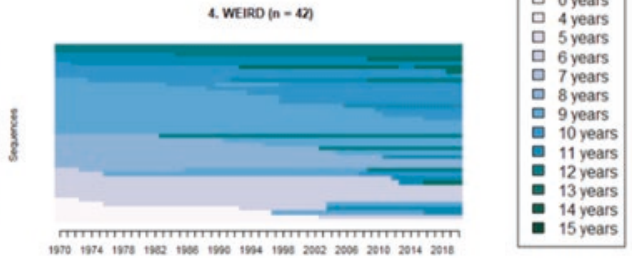

ree

Fig. 3.3 Sequences grouped by cultural spheres

when preprimary education becomes compulsory-policies high-income countries seem unwilling to introduce (GSP Digest 2019).

Regarding grouping by cultural spheres (Fig. 3.3), there are huge intracultural differences observable. In 'dominantly Muslim' countries, the durations range from five to twelve years from the 2000s onward. Given the theoretical framing of this edited volume (Chap. 1), one can detect a tendency toward likeness in culturally similar groups and concurrently, a resistance toward an increasing global isomorphism. This becomes evident from the stark differences regarding the duration in compulsory education between the 'WEIRD' and 'Catholic, Spanish-speaking' countries. In the latter, the general trend toward compulsory preprimary education is detectable through the very high durations of compulsory education, which in most cases means an earlier start to education rather than a later completion. Since UNESCO (2000) is largely propagating 
these models, vertical interdependencies seem to be an accelerator for that phenomenon. However, 'WEIRD' countries are largely holding back from implementing policies regarding preprimary education as mandatory.

Additionally, groupings by democratization level in the years 1970, 1992, 2000, or 2018 (not presented here) do not show similarities in trajectories. The grouped sequences are very diverse in their outset. Nevertheless, the duration of compulsory schooling per se might have a strong effect on democratization itself, making it an inverse relationship (as suggested by Paglayan 2020).

In sum, we can deduce partial evidence for countries in higher income groups having longer durations of compulsory education earlier. However, this does not manifest in a simplistic center, semi-periphery, and periphery manner. Aside from the intragroup differences, it is still interesting to see that the semi-periphery, operationalized through the upper middleincome group, exceeds a large portion of the high-income group at the end of the observation. Especially the surge of making preprimary education compulsory in South American countries contributes to the picture. Taking diffusion mechanisms seriously, I do not expect to observe perfect homogeneity in development paths. Indeed, the heterogeneity is detectable, especially in later years of the time frame. However, these differences go beyond what would be expected by a diffusion mechanism that assumes a one-sided direction from Western countries to others in the world. In other words, the norms of an ever-increasing grip on childhood-which decisively includes education for very young childrenovertook the former hegemonic WEIRD countries. In the future, one might expect to see the pattern develop as non-WEIRD countries adopt compulsory preprimary education first and through the interplay of horizontal and vertical interdependencies, the policy spreads around the globe. This direction is the opposite of what is observed within compulsory education in general (see Chap. 2). Lastly, it shows the investigation timing is relevant because the picture will probably look very different in 2030 or later. 


\section{Clustering the Trajectories}

For answering the question on how trajectories might be grouped together, it is now time to benefit from SQA as a metric analysis tool that searches for similar patterns across a multitude of sequences (Abbott 1995, 105). SQA makes it possible to compute distances between sequences and classify specific trajectories. The Optimal Matching (OM) algorithm lends itself perfectly for assessing dissimilarity. In a nutshell, the algorithm calculates which values of a sequence, when compared to another one, need to be changed to make the two sequences look similar. Thus, "an OM distance is the sum of two terms, a weighted sum of time shifts (indels) and a weighted sum of the mismatches (substitutions) remaining after the time shifts" (Studer and Ritschard 2016, 14). The algorithm goes through all the sequences and calculates the least 'costly' set of operations necessary to turn one sequence into another. Here, costs refer to values that have been assigned to operations, that is, the weights for every single operation, which get summarised in the end to define dissimilarity between sequences.

Since we are not only dealing with ordered values but also metric ones, I opted to define the weights for operations in a straightforward manner. A substitution of eight years of compulsory education with nine years costs 0.1 , substituting eight years with twelve years costs 0.4 . Thus, the costs are proportionate to the actual difference in duration of compulsory schooling. The only nonlinear substitution is present when a country has no compulsory education law at a given time, that is, they have a duration of zero years of compulsory education. Substituting this by any number of years has the overall maximum weight of 1.5 , thus representing the stark differences between having some form of mandatory education and having none at all. Studer and Ritschard (2016) report that by defining the costs for indel operations, researchers can influence the time sensitivity of the algorithm. High values render the distance measure very time sensitive because substitutions become increasingly cheaper than shifting sequences. Accordingly, I set the indel costs as low as mathematically meaningful to 0.8 so that the analysis would be as independent of small differences in the timing of policy enactment as possible. In the 
end, it is not my goal to overestimate time differences. For example, Indonesia and Syria both changed the duration of compulsory education from six to nine years; however, the former did so in 2003 and the latter in 2002. This one-year difference between them should not be more highly valued than the closeness in their actual overlapping trajectory.

Let us consider the example sequences of Mexico and Panama's durations of compulsory education from 1990 to 2010 in Fig. 3.4. Both countries start with a duration of six years of compulsory education but follow different paths after five observations. While the lower sequence (Panama) shows only one change in the state of the policy, that is, from six to eleven years, the one at the top (Mexico) shows a gradual increase regarding the duration of mandatory education from six to nine, and then eleven years. In this example, the algorithm, despite the low indel costs, opts for only substituting the values to calculate dissimilarity. For the period of 1993-1994, this means a sum of $0.3+0.3$ and for the period of 1995-2000, this means the sum of the difference of 0.2 for a duration of six years. The dissimilarity is thus: $0.3^{*} 2+0.2^{*} 6=1.8$.

The output of the OM calculation is a matrix that depicts the dissimilarity of countries' compulsory education law trajectories in the years 1970-2020. Using this matrix, I calculate clusters with the PAM (partitioning around medoids) algorithm as described by Kaufman and Rousseeuw (1990). The goal of this clustering algorithm is to assign each object to the nearest representative object. The representative object is defined "as that object of the cluster for which the average dissimilarity to all the objects of the cluster is minimal." (Kaufman and Rousseeuw 1990, 72). Although an elbow and silhouette test suggests four and two clusters, respectively, I opted, after several runs of the algorithm, for an

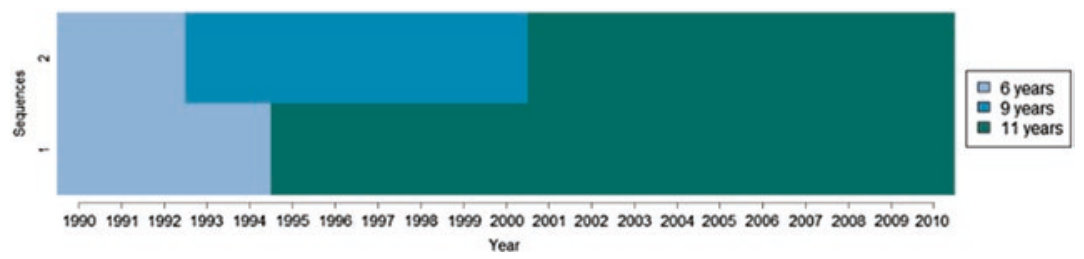

Fig. 3.4 Example sequences (Mexico and Panama from 1990-2010) 
eight-cluster solution. In a four-cluster solution, almost two-thirds of sequences would be part of one cluster, making the intracluster differences too high to analyze. Nevertheless, the tests show that I operate on rather slim margins. Holding true to the explorative goal of this chapter, this solution gives insights previously unseen. However, some intercluster differences are difficult to account for. Moreover, changing the time frame certainly changes the clustering. It is imperative to, again, stress that this chapter is not intended to draw causal relations or all-encompassing judgments from the clustering presented here. Nonetheless, we can generally take hints and insights from this endeavor.

Hence, the chosen solution produces clusters that are coherent in their trajectories and still downsized in a way that makes it possible to analyze and describe them. In Fig. 3.5, the trajectories of the eight distinct

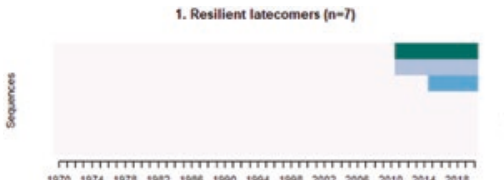

$1970 \quad 1974 \quad 1970 \quad 1902 \quad 1906 \quad 199019941990 \quad 2002 \quad 20062010 \quad 2014 \quad 2018$ Year
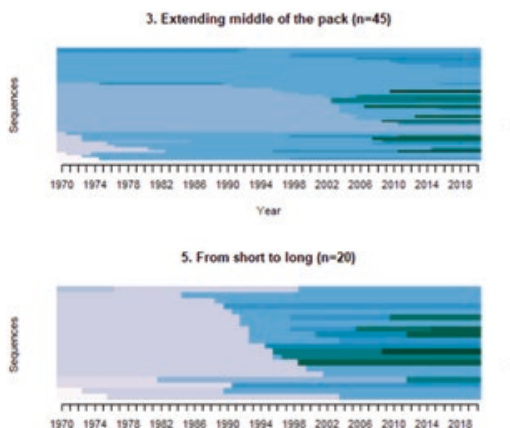

Year

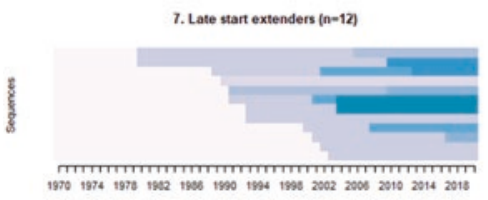
Year
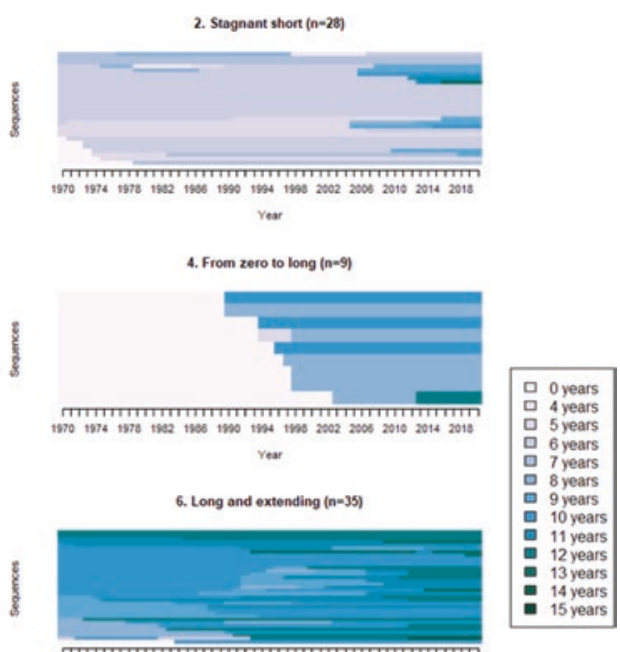

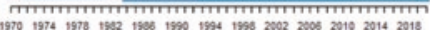
Year

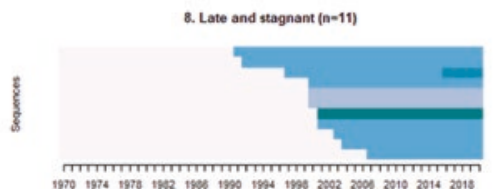

Year

Fig. 3.5 Sequences grouped by cluster 
clusters are shown. Next, I describe the different groups of trajectories separately.

\section{Resilient Latecomers (Cluster 1)}

The first cluster consists mostly of countries that did not introduce any compulsory education up until 2020. Although four countries introduced the policy very late and with large variation in durations, these are still part of the cluster. Similarity to one another for a long time, that is, having no compulsory education policy, is the key factor in building this cluster. After several years, we could expect the countries to become increasingly different in their durations, therefore, more similar to countries in other clusters. A large intragroup variety is visible, especially regarding countries that introduced any compulsion late. For instance, while Aruba introduced compulsory education with a duration of 13 years, Zambia’s first legislation mandates almost half of that.

\section{Stagnant Short (Cluster 2)}

Countries in this cluster started with short durations of compulsory education in 1970. After its introduction, there is a general tendency for an increase in the duration of compulsory education for some countries. The extensions are moderate and not as large as in the fifth cluster. However, this development began later in the 2000s, if at all. In the Philippines, six-year primary education was compulsory until 2011. In the same year, with the enactment of the K-12 reforms, secondary education was extended from four to six years and divided into two levels: four years of Junior High School and two years of Senior High School. All six years of secondary education are compulsory and free of charge for public schools. Additionally, one-year preprimary education became mandatory, which culminates to a total duration of thirteen years. In Rwanda, on the other hand, compulsory education started in 1962 with six years of primary school. A 1979 reform tried to extend primary school, but this did not hold for long and as ethnic tensions grew, the grades were essentially 
eliminated again. After the genocide, Rwanda followed quite a unique path and extended compulsory education via the '9-Year-Basic-Education Policy' in 2007 to extend mandatory education through senior secondary school which is still regarded as basic education (Mathisen 2012, 108-9).

\section{Extending Middle of the Pack (Cluster 3)}

Consisting of forty-five countries, this is the largest cluster by far and it naturally shows the largest intragroup differences when regarding it as cross sections for specific years. Concerning the trajectories, however, we see that it mostly starts with middle-range durations. While very few countries decrease the duration for a short period of time, most increase it starting as early as the 1970 s. The trajectories show a rather similar path: extensions of duration occur in two waves, at the end of the 1970s and around the 2010s. Especially during the latter wave, it seems that the duration of compulsory education converges toward rather long ones. For instance, the Dominican Republic shows the highest duration of compulsory education, that is, fifteen years in 2010. This was achieved by extending compulsion to three years of preprimary education. It now includes children from ages three to seventeen at the end of secondary education. The trajectory is similar to Ecuador's, in that both extend the durations gradually over time. This pattern of extending compulsory education toward younger children is also detectable in Brazil, Chile, and Costa Rica. Nonetheless, not all countries in cluster 3 end up with these high durations: While Bosnia and Herzegovina and Serbia extended compulsory education by one year in the 2000s, Croatia stayed with eight years throughout the time frame.

\section{From Zero to Long (Cluster 4)}

This particular cluster consists of countries with no compulsory education until roughly 1990 but then introduced long durations of ten to eleven years. While both Belize and Sudan introduced compulsory education in 1990 with a duration of eight years, Malawi started with five 
years of duration, which was then extended to eight years. The longest duration is mandated for children in Kenya. It was the last country in this cluster to introduce compulsion in 2008 but then became a frontrunner by extending the duration from eight to twelve years of education.

\section{From Short to Long (Cluster 5)}

Contrary to the previous cluster, these countries implemented compulsory education by 1970 . Starting in the mid to late 1980s, the previously short durations were extended, in some cases by a large margin reaching durations of up to fifteen years. The timing for these extensions is earlier than most countries within cluster 3. Paraguay and Argentina have similar paths; they both started with six years of compulsory education and extended the duration to nine, ten and eventually thirteen years, with Argentina extending to fourteen years. The largest difference in timing of these policies is only three years and while Paraguay was a bit earlier in the 1990s, Argentina is now quicker to extend. Another example of these steady extensions includes Turkey: When the Republic was founded in 1923, five years of primary education for six to eleven-year-olds became compulsory. From 1997 onward, compulsory education encompassed eight years of primary school, after which pupils could receive a secondary non-mandatory education at a four-year lyceum. "Another reform abolished the continuous primary school education and replaced it in 2012/2013 with a three-level, 12-year compulsory schooling divided into a 4-year primary school, a 4-year middle school, and a 4-year lyceum" (Karakaşoğlu and Tonbul 2015, 828).

Finally, a somewhat unconventional path becomes evident when looking at Thailand, where compulsory education was initially expanded in 1960 from four to seven years by extending the primary education by three years. However, after democratization in 1977, the system was rebuilt and the length of primary school was decreased by one year, which was added to upper secondary school, in effect decreasing compulsory education. Nevertheless, with the National Education Plan of 1999, lower secondary became compulsory, resulting in a duration of nine years. 


\section{Long and Extending (Cluster 6)}

Subsumed under the headline of this cluster are countries that already started with long durations of compulsory education in 1970. Almost all of them went on to extend these long durations further down the line. No waves of extensions can be detected. Moreover, those at the bottom in the 1970s converged toward the long durations of roughly fourteen years, showing a good exemplary case of uncoordinated betaconvergence. This holds especially true for the rather nonlinear trajectory Peru shows: In 1972, the first three years of secondary education were moved to basic education, which made it compulsory and extended the duration from six to ten years. However, this reform was abolished in the 1980s bringing the former structure back (Chuquilin Cubas 2011). Then, the constitution of 1993 declared education as compulsory for preprimary, primary, and secondary levels again (Marlow-Ferguson 2002, 1047). In the end, compulsion was extended to upper secondary education, resulting in fourteen years of compulsory education.

Another decrease in compulsory schooling happened in Azerbaijan, where after the fall of the USSR compulsory education law prescribed a nine years' duration, instead of the former ten years. Nevertheless, after some time, the duration was extended to eleven years in 2011. Interestingly, this new Education Law, making general secondary education mandatory, makes the Azerbaijani education system almost entirely conform to the principles of the Bologna process (International Bureau of Education 2011).

\section{Late Start Extenders (Cluster 7)}

Countries here start with no compulsory education, although these policies had been introduced in 2014 at the very latest. The trajectories are similar to those in cluster 4; however, the first introduction shows a rather short duration. Nonetheless, a decisive tendency for longer durations is detectable. While Cabo Verde extended compulsory education from six to ten years right away, Lebanon shows a stepwise extension from six to nine and finally ten years. Malaysia introduced compulsory education in 
2003 with a duration of six years and did not alter this policy. This holds true for Cameroon and Singapore as well. Given the discussions in Singapore before the introduction of compulsory education, described above, it is clear why the government might be hesitant to extend compulsory education, although the government initially advocated for a longer duration (Tan 2010).

\section{Late and Stagnant (Cluster 8)}

Similar to the previous cluster and the From zero to long cluster, countries here had no compulsory education in the 1970s. In most cases, there was no change since the introduction at the end of the 1990s. This is shown by the trajectories of Yemen and Mali in which a nine-year compulsory education was introduced in 1991 and 1992, respectively. These policies still stand today. In Qatar, on the other hand, compulsory education was introduced later in the Compulsory Education Law No. 25 of 2001 covering primary and secondary education that culminate in twelve years of education. Sri Lanka is the only member of this cluster that extended the duration of compulsory schooling by expanding compulsion through senior secondary school, that is, Grade 11.

\section{A Global Picture on Clusters of Compulsory Education Duration}

Finally, I look at the global picture of the duration of compulsory education. Figure 3.5 shows the difficulty of choosing the correct clustering method, since it remains unclear why some trajectories are part of one cluster and not another. Especially, some countries in cluster 3 (Extending middle of the pack) and cluster 6 (Long and extending) have very similarly looking trajectories. So, we need to review the global picture with the caveat that always comes with drawing strict distinctions where ranges would be more appropriate. When regarding a longer time frame, say from 1900 to today, I suspect the two mentioned clusters (depicted in purple and yellow) to be part of a similar supercluster. Nevertheless, we 
see subtle differences on average, so I urge readers not to draw conclusive absolutes of, for example, 'Canada is significantly different from the US', but rather, acknowledge differences in the developments of the duration of compulsory education, as subtle as they may be.

Moreover, there are several key elements that we can take away from this endeavor: As opposed to cross-sectional observations, examining trajectories helps highlight differences and similarities that would not have been visible before. Some countries followed a specific wave of either introducing or extending compulsory education starting from the 1990s until the mid-2000s. After that, the pace and margins of extensions increased, especially concerning countries that have had shorter durations of compulsory education. One first intuitive correlation might be traced back to international initiatives, especially the Education for All Framework for Action, which might have had a large impact on countries to either introduce or extend compulsory education.

Furthermore, the degree to which domestic factors vary in clusters is striking. For a comparison, I defined the dominant religion of a country in 1970 , in that at least $33 \%$ of the population is adherent to it as well as the Gender Rights variable used in building the cultural spheres. The latter is an index that combines the "Women's political empowerment index" (Sundström et al. 2015) and the "Exclusion by Gender Index" (Coppedge et al. 2019) from the Varieties of Democracy Project. The former takes its raw data from the World Religion Dataset (Maoz and Henderson 2013). Additionally, I investigated the percentage of highand low-income countries in the distinct clusters. Although there is at least one high-income country in every cluster, the highest percentages are in the Long and extending, the Extending middle of the pack and interestingly, also one in the cluster of Resilient latecomers. Furthermore, against first intuition, in the group of Resilient latecomers no country is defined as low income. In other clusters at least one low-income country can be found: in the From zero to long almost half of the countries and in the Late and stagnant cluster even more than half are low-income countries. Moreover, the Long and extending cluster shows the highest mean of the Gender Rights value, while the Late and stagnant countries show the lowest mean value. Interestingly, the Stagnant short countries have the second lowest mean gender value. Concerning the dominant religion 
there is no pattern discernible. In every cluster, at least half of the members have Christianity as a dominant religion, except the Resilient latecomers, where the percentage of countries is still over $40 \%$. The Extending middle of the pack with the Stagnant short shows the highest variability in dominant religions, being made up of Animist and Syncretic religions, Buddhism, Hinduism, Islam, Christianity, and nonreligious persons. Again, this shows that with rather broad domestic variables it is hardly possible to find correlations on compulsory education policy trajectories.

Additionally, when plotting the previously described cluster onto a world map (Fig. 3.6), it is astonishing to see that for some clusters a geographic pattern is hardly detectable. The largest cluster of the Extending middle of the pack is scattered throughout Southern, Central, and Eastern Europe, South America, and North Africa. A slight North-South difference can be noticed, which shows that some sub-Saharan as well as Southeast Asian countries are clustered together. Some striking evidence we can gather from this map is that there seems to be weak colonial

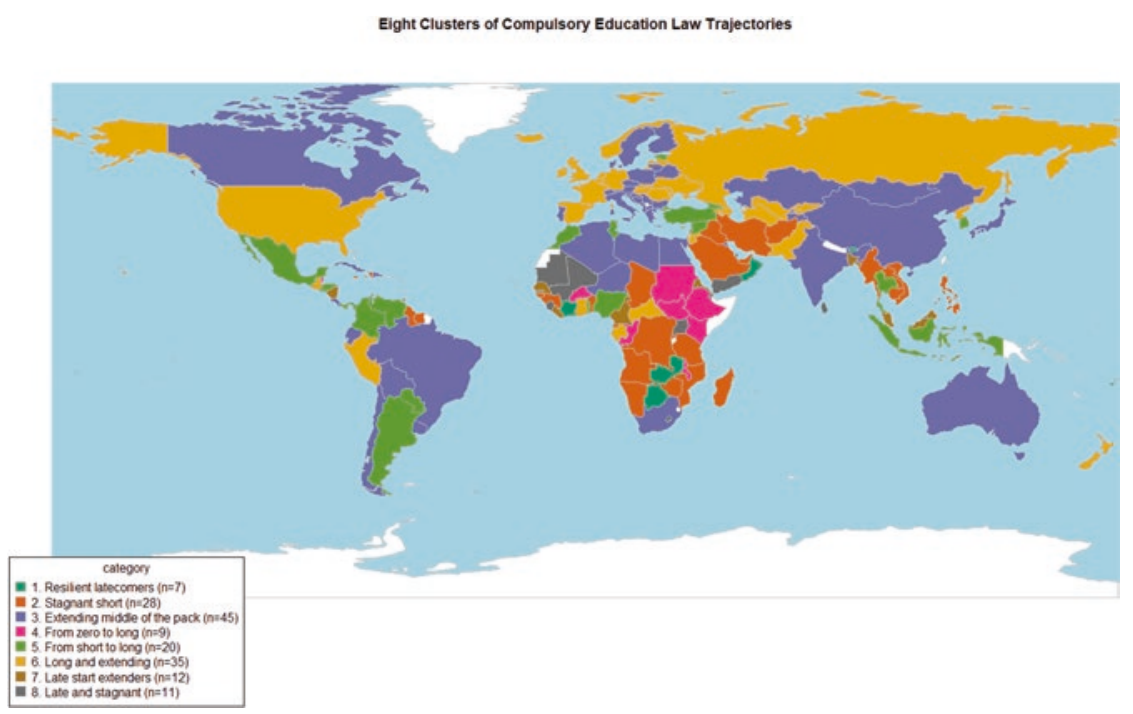

Fig. 3.6 World map showing countries' membership in clusters of trajectories of the duration of compulsory education 
heritage discernible, if any. The United Kingdom (UK), except in combination with South Africa, has a different compulsory education policy since 1970 than most of its former colonies. For example, India and former British colonies in sub-Saharan Africa not only differ in their trajectories with one another but also with the UK. This also holds true for other colonies of European states. Furthermore, after the breakup of the USSR, some countries seem to follow distinctly different paths than their former Union countries.

The goal of this chapter is not to draw conclusive or even causal statements about the development of compulsory education policy. However, what I can show is that international comparative education should refrain from 'easy' and overly hasty explanations. Nation-states as diverse as Belgium, Guatemala, and North Korea (Long and extending) show similarity regarding their trajectories of compulsory education policy. This could excite new ways of international comparative education research.

\section{Conclusion}

In this chapter, I started with the observation that recent international comparative education research has advanced at a rapid pace. Notions of an isomorphism of national education systems overshadow the actual analysis of different developmental paths of single elements of these systems. Past scholarship has been focused on the origins, output, or outcome of compulsory education legislation. In contrast, I argued to take into account the whole trajectories of education policy and use them as the focal point of research. This recontextualization in terms of environing policy changes in time and relation to other countries yields the possibility to simultaneously answer questions on 'what' policy change looks like as well as 'when' and 'in which order' this happens or does not happen.

With the help of sequence analysis tools, I demonstrated that when looking at the duration of compulsory education from 1970 until 2020, durations of compulsory education and their development paths show large differences that cannot be explained at first glance by crude 
classifications of economic development or cultural spheres. However, it is possible to generally discern, that higher income countries, on average, have longer durations of compulsory education and 'Catholic, Spanishspeaking' nation-states have high durations. The latter is probably due to a shift of norms regarding mandatory, preprimary education. It seems that we are witnessing a new diffusion dynamic in which policy innovation starts in non-Western states but transfers to the West in the future.

I set out to quantitatively cluster countries together based solely on their actual trajectories of compulsory education law. Eight clusters have been described, which, on the one hand, show remarkable overlap but, on the other hand, still have large intragroup variances. However, we can take hints from this clustering. For example, there are some regional specialties and, generally, higher income means earlier extension on average as well as longer durations of compulsory education in general. Furthermore, a second wave of introduction and extension of compulsory education seems to correlate-at least time-wise-with the increasing intensity of international discourses like Education for All or the Sustainable Development Goals, for example. While the first started at the World Conference on Education for All in Jomtien in 1990 and was renewed in the Dakar Framework for Action in 2000, the latter are currently shaping education policies around the world since 2015.

However, these are the first intuitions and do not claim causal relationships.

Moreover, accepting the clustering leads to imprecision when reviewing it as set-in-stone boundaries. Similarly, to what is argued in Chaps. 1 and 2 , the social reality of policy change shows overlaps that are changing throughout time and space. The clusters observed look different from ones built from sequences starting in 1900 and will look different when observed in 2050 .

Nonetheless, the information gathered here shows clearly the numerous, different paths, even though clustering proves difficult. Trying to discern broad trends in domestic factors is almost impossible. The precise interplay of political ideology, global discourses, and especially regional/ cultural discourses can have an impact on trajectories. This complexity is difficult to discern. However, with this descriptive investigation, I have found possible avenues where scholarship might be able to place further 
focus. In my view, this should motivate researchers to take a step back and take stock of the complexity found in the real world of education policies. It is tedious work but should nonetheless be done in order to understand what is really going on in a global education sphere that shows isomorphism only in a very abstract way.

Going forward, I suggest keeping the following in mind: To actualize complexity does not inhibit researching on a macro-level; rather, it should motivate us to do it more often in an all-encompassing way. Different and complex trajectories do not end in one telos but are instead open for debates situated in different locations and different times. The complexity of nation-states' education systems should be systematically described and analyzed before turning toward actual explanations that most probably will not yield one-size-fits-all solutions. I took a first step in that direction. Future research should take the insights presented in this chapter and expand on other constitutive parts of education systems. One should also focus on what determinants are influential in bringing about trajectories that unfold in a specific sequential order. Such an analysis should involve interdependencies between countries as well as national factors. A thorough and structured analysis could yield more insights into the global developmental paths of education systems. Furthermore, using this study as the starting point, new theories on the international transfer of education system characteristics could be possible. Taking a step back and considering the bigger picture helps in highlighting the shortcomings of research that global education policy needs to address.

\section{References}

Abbott, Andrew. 1992. From Causes to Events. Sociological Methods \& Research 20 (4): 428-455. https://doi.org/10.1177/0049124192020004002.

Abbott, Andrew. 1995. Sequence Analysis: New Methods for Old Ideas. Annual

Review of Sociology 21 (1): 93-113. https://doi.org/10.1146/ANNUREV. SO.21.080195.000521.

Anderson-Levitt, Kathryn M. 2003a. A World Culture of Schooling? In

Local Meanings, Global Schooling: Local Meanings, Global Schooling: Anthropology and World Culture Theory. New York, NY: Palgrave Macmillan. 
Accessed 3 November 2020. http:/www.loc.gov/catdir/description/ hol031/2002033307.html.

Anderson-Levitt, Kathryn M. 2003b. Anthropology and World Culture Theory. In Local Meanings, Global Schooling. New York, NY: Palgrave Macmillan. Accessed 3 November 2020. http:/www.loc.gov/catdir/description/ hol031/2002033307.html.

Arnove, Robert F. 2009. World-Systems Analysis and Comparative Education in the Age of Globalization. In International Handbook of Comparative Education, ed. Robert Cowen and Andreas M. Kazamias, 101-119. Dordrecht: Springer Netherlands.

Blanchard, Philippe. 2011. Sequence Analysis for Political Science. Committee on Concepts and Methods Working Paper (32). https://www.researchgate.net/ profile/Philippe_Blanchard3/publication/259444976_Sequence_Analysis_ for_Political_Science/links/0c96052ba2544e678e000000/SequenceAnalysis-for-Political-Science.pdf.

Boli, John, Francisco O. Ramirez, and John W. Meyer. 1985. Explaining the Origins and Expansion of Mass Education. Comparative Education Review 29 (2): $145-170$.

Busemeyer, Marius R., and Christine Trampusch. 2011. Review Article: Comparative Political Science and the Study of Education. British Journal of Political Science 41 (2): 413-443. https://doi.org/10.1017/ S0007123410000517.

Chuquilin Cubas, Jerson. 2011. La educación secundaria en Perú y sus profesores: cambios y continuidades. Revista Educación 35 (2). https://doi. org/10.15517/revedu.v35i2.461.

Cicek, Nazan. 2012. The Role of Mass Education in Nation-Building in the Ottoman Empire and the Turkish Republic, 1870-1930. In Mass Education and the Limits of State Building, c.1870-1930, ed. Laurence W.B. Brockliss, 224-250. Basingstoke: Palgrave Macmillan.

Coppedge, Michael, John Gerring, Carl H. Knutsen, Staffan I. Lindberg, Jan Teorell, David Altman, Michael Bernhard, et al. 2019. V-Dem Codebook v9: Varieties of Democracy (V-Dem) Project.

Courtin, Emilie, Vahe Nafilyan, Mauricio Avendano, Pierre Meneton, Lisa F. Berkman, Marcel Goldberg, Marie Zins, and Jennifer B. Dowd. 2019. Longer Schooling but Not Better Off? A Quasi-Experimental Study of the Effect of Compulsory Schooling on Biomarkers in France. Social Science \& Medicine 220: 379-386. 
Dale, Roger. 2000. Globalization and Education: Demonstrating a 'Common World Educational Culture' or Locating a 'Globally Structured Educational Agenda’? Educational Theory 50 (4): 427-448. https://doi.org/10.1111/ j.1741-5446.2000.00427.x.

Diaz-Serrano, Luis. 2020. The Duration of Compulsory Education and the Transition to Secondary Education: Panel Data Evidence from Low-Income Countries. International Journal of Educational Development 75: 102-189.

Dutta, Sumi S. 2019. Extension of the Right to Education Act Way too Costly, Proposal Set to be Shredded. The New Indian Express, November 28. Accessed 3 November 2020. https://www.newindianexpress.com/nation/2019/ nov/28/extension-of-the-right-to-education-act-way-too-costly-proposalset-to-be-shredded-2068281.html.

Gabadinho, Alexis, Gilbert Ritschard, Nicolas S. Müller, and Matthias Studer. 2011. Analyzing and Visualizing State Sequences in R with TraMineR. Journal of Statistical Software 40 (4): 1-37. https://doi.org/10.18637/JSS.V040.I04.

Griffiths, Tom G., and Robert Imre. 2013. Mass Education, Global Capital, and the World: The Theoretical Lenses of István Mészáros and Immanuel Wallerstein. New York, NY: Palgrave Macmillan.

GSP Digest. 2019. Global Social Policy Digest 19.1-2. Global Social Policy 19 (1-2): 159-178. https://doi.org/10.1177/1468018119846701.

Hodgson, David. 2016. What Do You Want to Do Your Life? Ethics and Compulsory Education. Journal of Education Policy 31 (4): 495-507. https:// doi.org/10.1080/02680939.2015.1119314.

Howlett, Michael, and Klaus H. Goetz. 2014. Introduction: Time, Temporality and Timescapes in Administration and Policy. International Review of Administrative Science 80 (3): 477-492. https://doi.org/10.1177/ 0020852314543210.

International Bureau of Education. 2011. World Data on Education: Seventh Edition 2010-11. Accessed 3 November 2020. http://www.ibe.unesco.org/ en/document/world-data-education-seventh-edition-2010-11.

Karakaşoğlu, Yasemin, and Yilmaz Tonbul. 2015. Turkey. In The Education Systems of Europe, ed. Wolfgang Hörner and Lutz R. Reuter, 2nd ed., 825-850. Cham: Springer.

Kaufman, Leonard, and Peter J. Rousseeuw. 1990. Finding Groups in Data: An Introduction to Cluster Analysis. Hoboken, NJ: Wiley-Interscience.

Landes, William M., and Lewis C. Solmon. 1972. Compulsory Schooling Legislation: An Economic Analysis of Law and Social Change in the Nineteenth Century. The Journal of Economic History 32 (1): 54-91. 
Maoz, Zeev, and Errol A. Henderson. 2013. The World Religion Dataset, 1945-2010: Logic, Estimates, and Trends. International Interactions 39: 265-291.

Marlow-Ferguson, Rebecca. 2002. World Education Encyclopedia: A Survey of Educational Systems Worldwide. 2nd ed. Detroit: Gale Group.

Mathisen, Jay. 2012. Education Reform in Rwanda: Impacts of Genocide and Reconstruction on School Systems. Doctor of Education (EdD) 11. http:// digitalcommons.georgefox.edu/edd/11.

Meyer, John W., John Boli, George M. Thomas, and Francisco O. Ramirez. 1997. World Society and the Nation-State. American Journal of Sociology 103 (1): 144-181. https://doi.org/10.1086/231174.

Murtin, Fabrice, and Martina Viarengo. 2011. The Expansion and Convergence of Compulsory Schooling in Western Europe, 1950-2000. Economica 78 (311): 501-522. https://doi.org/10.1111/j.1468-0335.2009.00840.x.

Paglayan, Augustina. 2020. The Non-Democratic Roots of Mass Education: Evidence from 200 Years. American Political Science Review, 1-20. https:// doi.org/10.1017/S0003055420000647.

Pierson, Paul. 2004. Politics in Time: History, Institutions, and Social Analysis. Princeton and Oxford: Princeton University Press.

Ramirez, Francisco O. 2013. Reconstituting Children: Extension of Personhood and Citizenship: Francisco O. Ramirez. In Age Structuring in Comparative Perspective, ed. David I. Kertzer and K.W. Schaie, 143-165. Hoboken: Taylor and Francis.

Rickenbacker, William F. 1999. The Twelve-Year Sentence: Radical Views of Compulsory Schooling. San Francisco: Fox \& Wilkes.

Stephens, Melvin, Jr., and Dou-Yan Yang. 2014. Compulsory Education and the Benefits of Schooling. American Economic Review 104 (6): 1777-1792.

Strang, David, and John W. Meyer. 1993. Institutional Conditions for Diffusion. Theory and Society 22 (4): 487-511.

Studer, Matthias, and Gilbert Ritschard. 2016. What Matters in Differences between Life Trajectories: A Comparative Review of Sequence Dissimilarity Measures. Journal of the Royal Statistical Society: Series A (Statistics in Society) 179 (2): 481-511. https://doi.org/10.1111/RSSA.12125.

Sundström, Aksel, Pamela Paxton, Yi-ting Wang, and Staffan I. Lindberg. 2015. Women's Political Empowerment: A New Global Index, 1900-2012. The Varieties of Democracy Institute Working Paper 19. https://www.v-dem.net/ media/filer_public/27/ef/27efa648-e81e-475a-b2df-8391dc7c840b/v-dem_ working_paper_2015_19.pdf. 
Takayama, Keita. 2015. Provincialising the World Culture Theory Debate: Critical Insights from a Margin. Globalisation, Societies and Education 13 (1): 34-57. https://doi.org/10.1080/14767724.2014.967485.

Tan, Jason. 2010. Compulsory Education in Singapore-Who Benefits? Asia Pacific Journal of Education 30 (4): 401-418. https://doi.org/10.108 0/02188791.2010.520203.

UN General Assembly. 1948. Universal Declaration of Human Rights. 1948.

UNESCO. 2000. Dakar Framework for Action: Education for All. Meeting

Our Collective Commitments. Accessed 3 November 2020. https://sustainabledevelopment.un.org/content/documents/1681Dakar\%20Framework\% 20for\%20Action.pdf.

Verger, Antoni, Clara Fontdevila, and Adrián Zancajo. 2016. The Privatization of Education: A Political Economy of Global Education Reform. New York, NY: Teachers College Press.

Wallerstein, Immanuel M. 2005. World-Systems Analysis: An Introduction. 4th Print. Durham: Duke University Press.

Wilson, Tanya. 2017. Compulsory Education and Teenage Motherhood. Accessed 3 November 2020. http://hdl.handle.net/1893/25614.

Zhang, Tiedao, and Zhao Minxia. 2006. Universalizing Nine-Year Compulsory

Education for Poverty Reduction in Rural China. International Review of Education 52 (3-4): 261-286. https://doi.org/10.1007/s11159-006-0011-z.

Open Access This chapter is licensed under the terms of the Creative Commons Attribution 4.0 International License (http://creativecommons.org/licenses/ by/4.0/), which permits use, sharing, adaptation, distribution and reproduction in any medium or format, as long as you give appropriate credit to the original author(s) and the source, provide a link to the Creative Commons licence and indicate if changes were made.

The images or other third party material in this chapter are included in the chapter's Creative Commons licence, unless indicated otherwise in a credit line to the material. If material is not included in the chapter's Creative Commons licence and your intended use is not permitted by statutory regulation or exceeds the permitted use, you will need to obtain permission directly from the copyright holder.

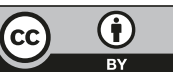

